\title{
A LOW-POWER, LOW-LEAKAGE, BI-STABLE PLANAR ELECTROLYSIS MICRO GATE VALVE
}

\author{
Jeremy A. Frank and Albert P. Pisano \\ Berkeley Sensor \& Actuator Center, Department of Mechanical Engineering \\ University of California, Berkeley, California 94720-1774
}

\begin{abstract}
The micro gate valve presented in this paper demonstrates improved sealing over previous planar silicon valves [1]. This is achieved using a wafer-level, five-mask fabrication process that removes the leakage paths above and below the moving gate, a problem that plagued earlier valve versions. Current valve designs exhibit closed fluidic resistances as high as $3.9 \times 10^{15} \mathrm{Ns} / \mathrm{m}^{5}$ with fluidic-resistance ratios greater than 3,500. This valve uses lowpower electrolysis of the working fluid for actuation, making it suitable for portable microfluidic applications where battery energy is limited. These valves require as little as $9 \mu \mathrm{J}(0.23 \mu \mathrm{W})$ to open and $22 \mu \mathrm{J}(0.6 \mu \mathrm{W})$ to close at $5.0 \mathrm{~V}$. No energy is required to maintain the state of the valve. The inert nature of the silicon and Pyrex $^{\circledR}$ fabrication materials also makes this valve suitable for high-temperature and corrosive environments in addition to the biological applications discussed here.
\end{abstract}

\section{INTRODUCTION}

This microvalve is designed for integration into a portable bioassay microfluidic system where all of the energy and working fluid are stored on board the device. Thus, the valves are designed to be both low leak and low power as well as straightforward to fabricate and easy to integrate with other fluidic components to form complete microfluidic bioassay systems.

Portable microfluidic devices have a very limited supply of working fluid and the use of this fluid must be tightly budgeted to increase the life of the system. The microvalves used for these applications must have low leakage rates to increase the precision with which fluid is distributed to other parts of the system. Large leakage rates waste more fluid, decrease system life, and reduce the overall performance of the system as the leakage will induce uncertainty in the position of fluid relative to assay chambers and sensors. This does not mean that a valve must possess an infinite closed fluidic resistance to be functional. While there are applications for very well sealing valves [2], the delivery of both $100 \mathrm{mg} / \mathrm{dL}$ and $500 \mathrm{mg} / \mathrm{dL}$ concentrations of insulin to an adult could be controlled by a valve with a fluidic-resistance ratio of 3,500 .

Additionally, due to the meager amount of energy available from the system battery, the valves are required to have low power consumption. The energy consumption of the valves is designed to make up only a fraction of that deliverable by the system battery, ensuring that the majority of the energy budget can be allocated to sensing and drug/antidote delivery.

Electrolysis actuation is chosen because of the low-power nature of electrolysis. The bi-stable valve would seal equally as well under thermal-bubble or electrostatic actuation because the sealing force is due to the suspension and valve seat geometry and not the specific actuation mechanism. The actual means of actuation would be defined by other system parameters such as power budget and operation speed.

The valve design is also planar, making it easy to integrate with other planar components such as mixers and pumps. Planar systems offer the potential for reduced dead volume within the device, as components can be placed adjacent to one another, obviating any large-volume through-wafer fluidic passages.
Additionally, the inert nature of the materials comprising the valve, Pyrex ${ }^{\circledR}$, silicon and platinum, suggest that the valve is also suitable for long-term use as well as in high-temperature and corrosive scenarios.

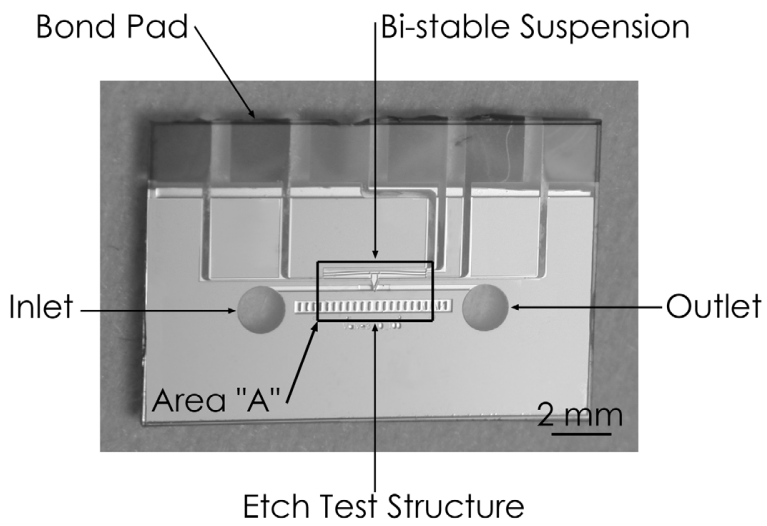

Figure 1. A photo of the top view of the valve, looking through the Pyrex ${ }^{\circledR}$ cover wafer. Visible are the inlet, outlet, bi-stable suspension, platinum bond pads and a test structure used to monitor lateral silicon etch progression. Area " $A$ " is enlarged in Fig. 2.

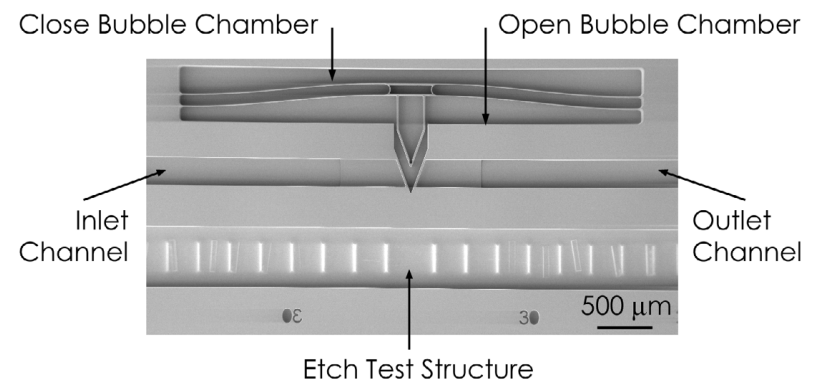

Figure 2. Area "A" of Fig. 1 shows a close-up picture of the bistable suspension for a valve in the open position. Visible are the inlet and outlet channels, close and open bubble chambers and the lateral etch test structure.

\section{VALVE DESIGN AND FABRICATION}

Each valve consists of an inlet, outlet and movable gate etched into the device layer of an SOI wafer, Fig. 1. The fluidic channels etched in the silicon are capped with a patterned Pyrex ${ }^{\circledR}$ wafer that is anodically bonded to the silicon surface. The actuating platinum electrodes are evaporated onto the surface of the Pyrex ${ }^{\mathbb{B}}$ and are located above the bubble-actuation chambers. The gate obstructs the fluid flow when the valve is closed and is anchored to the surrounding silicon by a bi-stable suspension. This suspension maintains the state of the valve by holding the gate open or closed, Fig. 2. Thus, no power is required to hold the state of the valve, reducing the overall energy consumption. In addition, the suspension is necessary to prevent loss or misalignment during fabrication. Previous planar valve designs had problems with the loss of free-floating parts during fabrication [3]. 


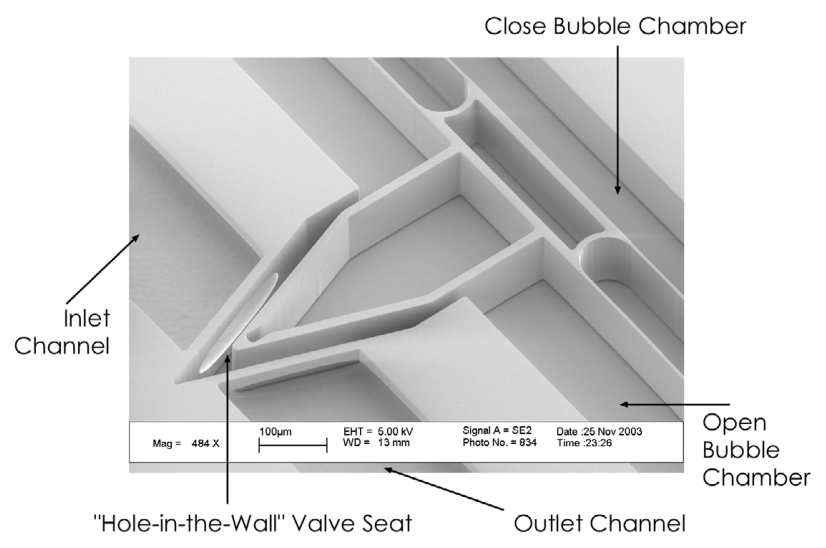

Figure 3. SEM showing the valve seat. Valve is in the open position. Visible are the inlet and outlet channels, close and open bubble actuation chambers and the valve seat fabricated with the "hole-in-the-wall" process.

Fluid must flow through a hole etched in the center of the sidewall of a channel, Fig. 3. This hole creates a valve seat that completely encompasses the flow, reducing leakage above and below the gate when the valve is closed. The hole is the key artifact of the fabrication process, Fig. 4, and is responsible for the improved sealing.

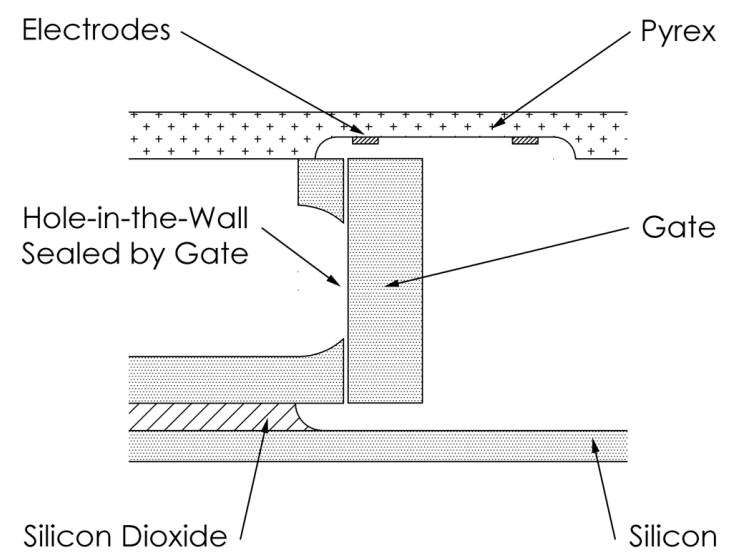

Figure 4. Cross-sectional view of the valve seat shows how the gate can be brought in contact with the seat, removing the leakage paths above and below the gate.

The valve is actuated with bubbles of hydrogen and oxygen created by electrolysis of the working fluid, deionized water. Bubbles are grown in the appropriate chamber and apply increasing pressure to the suspension members, eventually flipping the suspension and changing the state of the valve, Figs. 5, 6. The platinum metal used in the electrodes is necessary to catalyze the reaction of the hydrogen and oxygen gas to water, thereby collapsing the bubbles. This helps remove the bubbles from the chamber and allows the valve state to be changed at an increased rate.

The fabrication process is similar to that detailed in [4]. It uses a five-mask, wafer-level process that is typical of the hole-inthe-wall fabrication method. Trenches of two different depths are etched in the device layer of a silicon-on-insulator wafer $(100 \mu \mathrm{m}$ device layer, $4 \mu \mathrm{m}$ buried oxide). Deep trenches are etched to the buried oxide and serve as the majority of the fluidic pathways. The shallow channels are necessary to fabricate the valve seat.
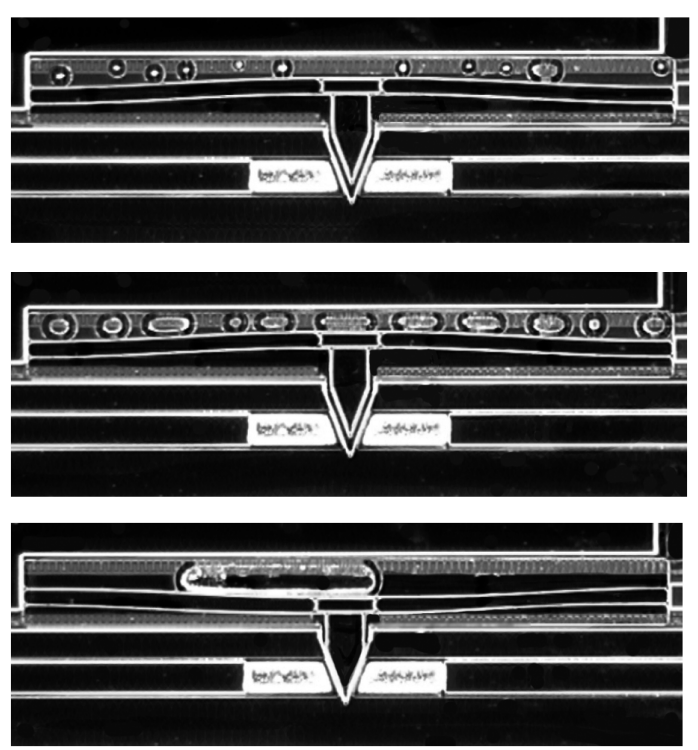

Figure 5. Sequential photos show the closing process. Bubbles of hydrogen and oxygen grown in the close bubble chamber apply increasing pressure to the bi-stable suspension that flips the gate and closes the valve.
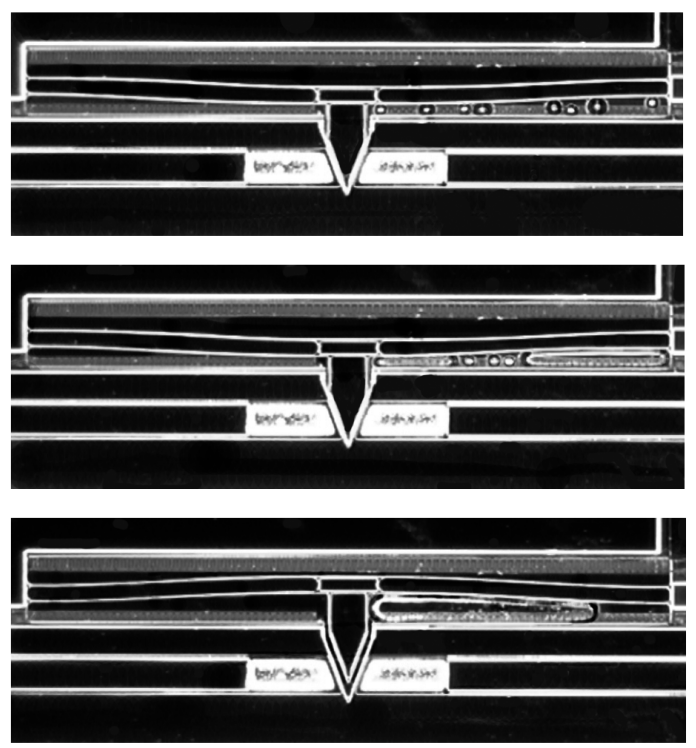

Figure 6. Sequential photos show the opening process. It was discovered that only one of the two opening bubble chambers was necessary to open the valve. This reduces the energy consumption of the valve.

All exposed silicon is then passivated in a wet oxidation step that performs two functions: it protects the silicon during the subsequent isotropic etch step and it serves as an oxide polish step that removes the DRIE scallops from the valve seat positioned in the side wall of the channel and improves sealing.

The oxide is removed from the bottom of the shallow channels using a directed plasma etch, exposing the silicon. The buried oxide layer is thick enough to remain following this step.

An isotropic plasma etch of silicon is performed which etches laterally beyond the oxide passivation of the shallow channels, puncturing the thin wall and forming the valve seat, Fig. 7. 


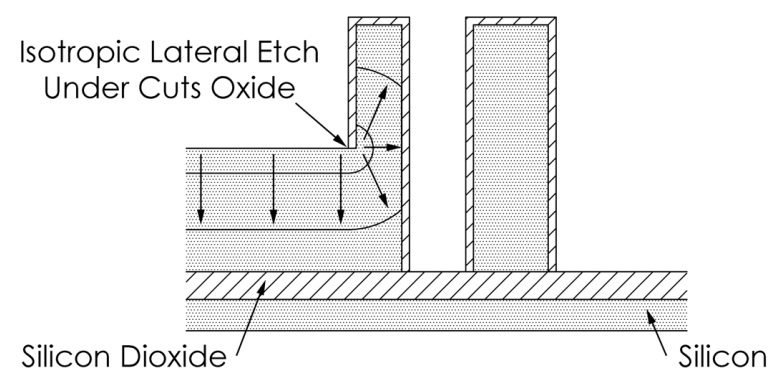

Figure 7. The isotropic silicon etch progresses laterally under the oxide passivation, puncturing the silicon wall and forming the valve seat.

The fifth mask is used to define backside through-wafer interconnects that can be omitted if in-plane interconnects are used [5].

The silicon wafer is then subjected to an isotropic HF acid etch to remove all of the passivating oxide to release the moving parts and prepare for the anodic bond.

The capping Pyrex ${ }^{\circledR}$ wafer is patterned with reliefs to ensure that the moving parts of each valve do not become bonded to the Pyrex $^{\circledR}$. Platinum (titanium adhesion layer) is evaporated onto the Pyrex $^{\circledR}$ and patterned using a lift-off technique.

The Pyrex ${ }^{\circledR}$ wafer is aligned and anodically bonded to the silicon wafer. The wafer stack is then diced following a scheme detailed in [6] that ensures that the microfluidic channels are not contaminated with the cooling water of the dicing saw. Additionally, portions of the silicon can then be broken off to expose the bond pads on the Pyrex ${ }^{\circledR}$ for the open and close electrodes.

The progression of the isotropic lateral etch is of paramount concern during the fabrication as it will define the final geometry of the orifice. This orifice geometry must be tightly controlled if the performance of valves fabricated on different wafers is to be the same. A test structure has been developed that allows for insitu monitoring of the lateral etch, allowing one-micron resolution of the etch progression, Fig. 8 .

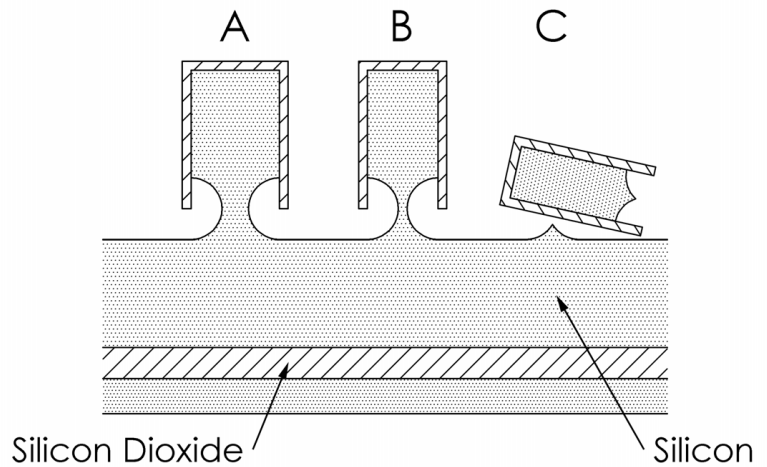

Figure 8. Mesas A, B, and $C$ are of decreasing thickness. The lateral etch progression is monitored by the fall of the mesas.

Mesas of differing widths are etched during the shallowtrench etch in the fabrication process. These mesas become undercut during the isotropic silicon etch and topple sequentially, indicating the extent of the lateral etch. Using these test structures, it has been shown that the lateral etch differs only by $+/-1$ micron across the wafer which results in a difference in orifice height of $+/-4 \%$. This is important as a basic analysis done in [4] suggests that there is an optimum orifice height that can increase the fluidicresistance ratio by a factor of 10 or more.

\section{EXPERIMENTAL SETUP}

The fluidic resistance of the valves was measured using a syringe pump and a pressure sensor, Fig. 9. The pressure sensor measured the pressure drop across the valve for a range of volumetric flow rates of deionized water set by the syringe pump. This experiment was performed for both the open and closed states of the valve, Figs. 10-12. Additionally, actuation time as a function of energy consumption was investigated, Fig. 13.

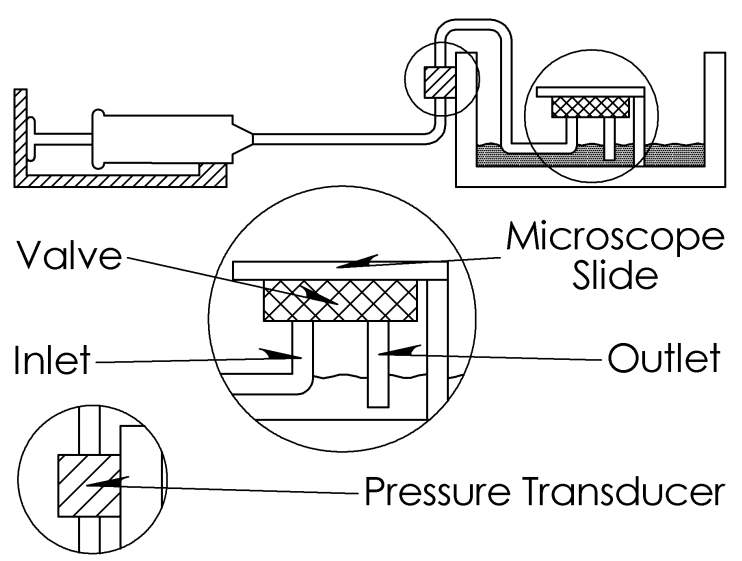

Figure 9. The valves were tested in an experimental setup like the one shown here. The outlet of the valve was submerged in water to ensure that the outlet pressure was not affected by evaporation or dripping of the exiting fluid.

\section{RESULTS AND DISCUSSION}

The closed and opened fluidic resistance for one of the fabricated valves is presented in Figs. 10 and 11. Data was taken for 12 actuations over a period of 96 hours. The fluidic capacitance of the testing setup greatly increased the time to equilibrium of the pressure drop across the valve for a set flow rate.

The actuation time of the valve was dependent upon the amount of energy used to close the valves. Greater energy was required for faster actuation times as the gas generation rate is proportional to the current flowing between the electrodes. The valves took as little as $22 \mu \mathrm{J}$ to close and $9 \mu \mathrm{J}$ to open at $5.0 \mathrm{~V}$. It was noticed that the actuation of only one open bubble chamber was necessary to open the valve, a discovery that reduced the energy consumption of the valve.

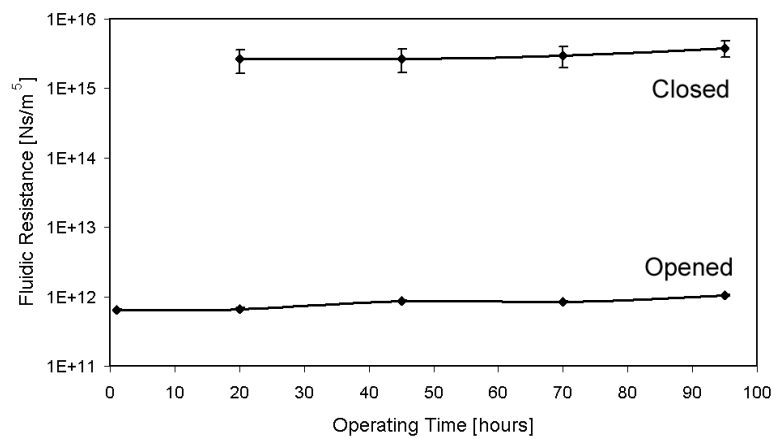

Figure 10. The fluidic resistance for one valve in both the opened and closed states over time. Data is given for a 95\% confidence interval. 


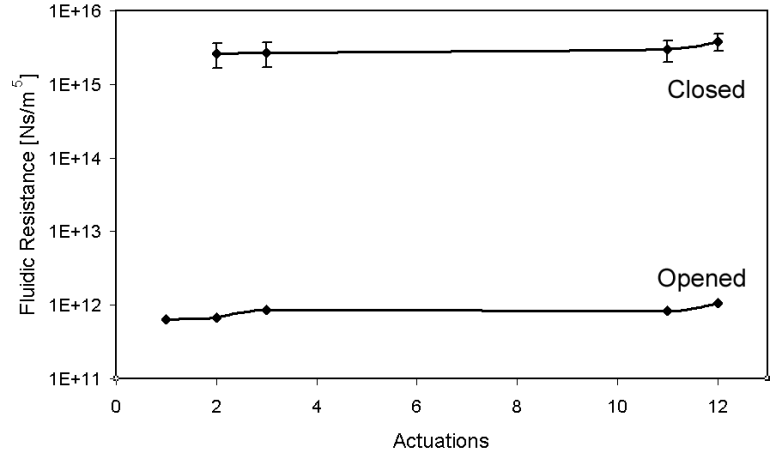

Figure 11. The fluidic resistance for the same valve as a function of the number of actuations.

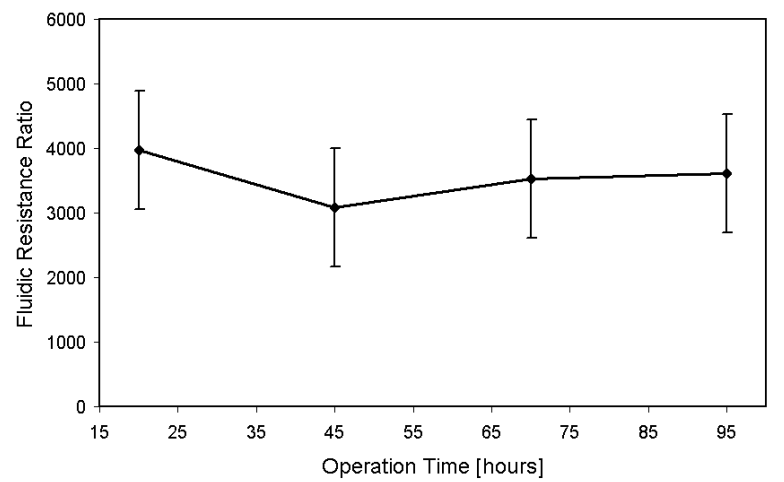

Figure 12. The fluidic-resistance ratio of the valve remained fairly steady over the duration of the tests. Data is given for a 95\% confidence interval.

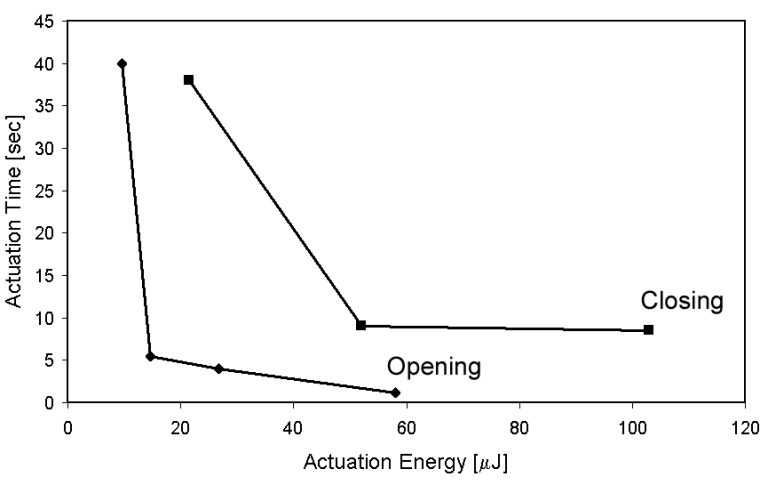

Figure 13. Actuation energy is inversely proportional to the actuation time. Energy consumption as low as $9 \mu J$ was achieved for an actuation time of 40 seconds $(0.23 \mu \mathrm{W})$. An actuation time of 1.2 seconds was achieved for an energy consumption of $58 \mu \mathrm{J}$ $(48 \mu W)$.

The data shows that the fluidic resistance of the valve differs by $25 \%$ over the length of the tests. This variation is most likely due to a misalignment between the wedge-shaped gate and the seat. The valve design uses a large safety factor in the bi-stable suspension design to ensure that the valve closes under the pressure available from the electrolysis bubble [7]. This results in long, thin beams $(3 \mathrm{~mm})$ that are compliant enough to allow for rotation of the gate during actuation. Thus, one side of the gate makes contact with the seat before the other and it is conceivable that the overly weak bi-stable suspension is not sufficiently forceful to guide the gate completely home. This design flaw is easily repairable in future versions of the valve.

\section{CONCLUSION}

A planar gate valve fabricated with the hole-in-the-wall process has been shown to have acceptable fluidic resistance and fluidic-resistance ratios for microfluidic applications. The fabrication process is wafer-level and easily allows for the integration of the valve with other planar components. This helps reduce the dead volume of the system, as components can be placed adjacent to one another.

The hole-in-the-wall fabrication process removes the leakage paths above and below the moving gate that plagued earlier planar valve designs, improving performance. The bi-stable suspension reduces the energy consumption of the valve by allowing the valve to only require power to change state. This bi-stable suspension also enables wafer-level fabrication, as the moving parts remain tethered to the substrate during fabrication. Thus, no free-moving parts are lost in the process as in previous planar examples.

Closed fluidic resistances of up to $3.9 \times 10^{15} \mathrm{Ns} / \mathrm{m}^{5}$ with fluidic-resistance ratios greater than 3,500 have been achieved. Energy and power consumptions were shown to be as low as $9 \mu \mathrm{J}$ $(0.23 \mu \mathrm{W})$ to open and $22 \mu \mathrm{J}(0.6 \mu \mathrm{W})$ to close at $5.0 \mathrm{~V}$. Shorter actuation times of 10 seconds to close can be achieved with an increase in power consumption to $48 \mu \mathrm{W}$.

Travel support has been generously provided by the Transducers Research Foundation and by the DARPA MEMS and DARPA BioFlips programs.

\section{REFERENCES}

1. A.P. Papavasiliou, A.P. Pisano, and D. Liepmann, "High-Speed and Bi-Stable Electrolysis-Bubble Actuated Gate Valves", Technical Digest of TRANSDUCERS '01, Munich, Germany, 6/1013/01, Transducer Research Foundation, Cleveland (2001), pp. 940-43.

2. J. Xie, X. Yang, X. Wang, and Y.C. Tai, "Surface Micomachined Leakage Proof Parylene Check Valve", Technical Digest MEMS 2001, $14^{\text {th }}$ IEEE International Conference on Micro Electro Mechanical Systems, Interlaken, Switzerland, 1/21-25/01, IEEE (2001), pp. 539-42.

3. A.A. Deshmukh, D. Liepmann, and A.P. Pisano, "Characterization of a Micro-Mixing, Pumping and Valving System”, Technical Digest of TRANSDUCERS '01, Munich, Germany, 6/10-13/01, Transducer Research Foundation, Cleveland (2001), pp. 950-53.

4. J.A. Frank and A.P. Pisano, "Low-Leakage Micro Gate Valves", Technical Digest of TRANSDUCERS '03, Boston, MA, 6/8-12/03, Transducer Research Foundation, Cleveland (2003), pp. 143-46.

5. S. Zimmermann, J.A. Frank, D. Liepmann, and A.P. Pisano, "A Planar Micropump Utilizing Thermopneumatic Actuation and InPlane Flap Valves", Technical Digest MEMS 2004, $17^{\text {th }}$ IEEE International Conference on Micro Electro Mechanical Systems, Maastricht, The Netherlands, 01/25-29/04, IEEE (2004), pp. 46265

6. S. Zimmermann, D. Fienbork, B. Stoeber, A.W. Flounders, D. Liepmann, "A Microneedle-Based Continous Glucose Monitor: Fabricated on a Wafer-Level Using In-Device Enzyme Immobilization", Technical Digest of TRANSDUCERS '03, Boston, MA, 6/8-12/03, Transducer Research Foundation, Cleveland (2003), pp. 99-102.

7. J. Qiu, J.H. Lang, A.H. Slocum, "A Centrally-Clamped Parallel-Beam Bistable MEMS Mechanism”, Technical Digest MEMS 2001, $14^{\text {th }}$ IEEE International Conference on Micro Electro Mechanical Systems, Interlaken, Switzerland, 1/21-25/01, IEEE (2001), pp. 353-5. 